erschienen in: Karl H. Hörning/Julia Reuter (Hrsg.), Doing Culture. Neue Positionen zum Verhältnis von Kultur und sozialer Praxis, Bielefeld: Transcript Verlag, S. 108-126

\title{
Regelmäßigkeit und Regelhaftigkeit. Die Abschirmung des technischen Kerns als Leistung der Praxis
}

Ingo Schulz-Schaeffer, Institut für Soziologie, TU Berlin, Franklinstr. 28/29 (Sekr. FR 2-5), D-10587 Berlin, email: schulz-schaeffer@tu-berlin.de

\section{Einleitung}

Der gemeinsame Nenner praxistheoretischer Ansätze besteht darin, dass sie sich für Regelmäßigkeiten sozialen Handelns interessieren, die nicht Ausdruck einer bewusst regelhaften Handlungsorientierung sind. Praxistheorie ist die Theorie jener sozialen Mechanismen, die Akteure in die Lage versetzen und dazu bringen, "unabsichtlich und ohne bewußte Befolgung einer ausdrücklich als solcher postulierten Regel sinnvolle und geregelte Praktiken hervorzubringen" (Bourdieu 1992: 99; vgl. ders. 1979: 215). Es ist die Theorie der inkorporierten und impliziten Wahrnehmungs-, Denk- und Handlungsschemata, die die Akteure in einer ihnen selbstverständlichen, routinisierten und nicht weiter reflektionsbedürftigen Weise befähigt, auf die praktischen Anforderungen der Handlungsfelder, auf die diese Schemata bezogen sind, angemessen zu reagieren.

Mit der praxistheoretischen Herangehensweise verbindet sich deshalb eine doppelte Regelskepsis: Zum einen ein Vorbehalt gegen sozialwissenschaftliche Erklärungen, die auf regelhaften Beschreibungen der RegelmäBigkeiten des sozialen Geschehens beruhen, also gegen das Vorgehen, "die soziale Welt in der Sprache der Regel zu beschreiben und so zu tun, als habe man die sozialen Praktiken erklärt, wenn man die explizite Regel benannt hatte, nach der sie angeblich hervorgebracht werden" (Bourdieu 1992: 99). Zum anderen eine deutliche Skepsis gegen die Annahme, dass dort, wo in der sozialen Welt selbst explizite Regeln geltend gemacht werden, es die Befolgung dieser Regeln ist, die die beobachteten Regelmäßigkeiten des Handelns hervorbringen - verbunden mit der Vermutung, dass es auch in diesem Fall eher die implizite Logik des praktischen Wissens und Könnens ist, die das Geschehen tatsächlich strukturiert.

Vor diesem Hintergrund soll es im Folgenden um die Frage des Verhältnisses der impliziten Logik der Praxis und der expliziten Logik der Befolgung kodifizierter Regeln gehen. Dazu werde ich in einem ersten Schritt kurz auf die bei Bourdieu aus der Regelskepsis geborene praxistheoretische 
Erklärung von Regelmäßigkeiten des Handelns eingehen (2.). Ich werde dann auf eine charakteristische Eigenschaft der impliziten Logik der Praxis hinweisen: auf den Umstand, dass es sich um eine Logik des Unscharfen und Ungefähren handelt. Dieser Umstand führt zu der Folgerung, dass es Situationen geben kann, in denen es im Interesse der Erwartungssicherheit sinnvoll oder erforderlich sein kann, von habituell hervorgebrachtem auf regelgeleitetes Verhalten umzustellen (3.). Anschließend soll betrachtet werden, unter welchen Bedingungen explizite Regeln die Chance besitzen verhaltenswirksam zu werden (4.). Die Mechanismen, die hier von Bedeutung sind, konstituieren das, was ich als den technischen Kern eines RegelWirkungs-Zusammenhangs bezeichne. Die Wirksamkeit expliziter Regeln hängt dennoch nicht allein von diesem technischen Kern ab, sondern gleichzeitig davon, in welchem Ausmaß es gelingt, ihn gegenüber externen Einflüssen abzuschirmen. Und das, so wird sich zeigen, ist nichtreduzierbar eine Leistung praktischen Wissens und Könnens (5.). Abschließend werden einige verallgemeinernde Folgerungen aus diesen Befunden für das Verhältnis zwischen den regelhaften Abläufen regelgeleiteten Verhaltens und den Regelmäßigkeiten praktischen Handelns gezogen (6.).

\section{Regelskepsis}

Besonders deutlich ausgeprägt finden sich beide Formen der Regelskepsis in der Praxistheorie Pierre Bourdieus. Die Ablehnung regelhafter Beschreibungen zur Erklärung der Regelmäßigkeiten des sozialen Geschehens bildet geradezu die Negativfolie für die Ausarbeitung seiner Praxistheorie. Diese, von ihm als als "Objektivismus" (Bourdieu 1987: 51), "Juridismus" (ebd.: 75) oder auch als "legalistische(r) Formalismus" (Bourdieu 1979: 207) bezeichnete Vorgehensweise galt es, so Bourdieu, "zu bekämpfen [...], um jene Theorie entwickeln zu können" (Bourdieu 1992: 110).

Der Objektivismus, das ist Bourdieus Kernargument, begeht "eine(n) der unheilsvollsten Fehlschlüsse in den Humanwissenschaften" (Bourdieu 1992: 81): Den Fehlschluss, die theoretische Sicht auf die Dinge in die Dinge selbst zu projizieren (vgl. Bourdieu 1981: 305; 1987: 148). Er unterstellt, dass die explizierten Regeln, die die beobachteten Regelmäßigkeiten der Praktiken beschreiben, zugleich auch die Erzeugungsgrundlage dieser Praktiken sei (Bourdieu 1987: 28, 71). Dass dies ganz und gar nicht der Fall ist, ist eine der zentralen Aussagen des Habitusbegriffs. Denn "[a]ls einverleibte, zur Natur gewordene und damit als solche vergessene Geschichte" (ebd.: 
105) beruhen die Dispositionen des Habitus eben gerade nicht auf einem bewussten Regelwissen, sondern auf der stillschweigenden Selbstverständlichkeit derjenigen Wahrnehmungs-, Denk- und Handlungsschemata, die den jeweiligen Habitus bilden. Deren Wirksamkeit wird auch nicht schon dadurch außer Kraft gesetzt, dass die Akteure selbst explizite, regelhafte Vorstellungen ihrer Praxis entwickeln. Jedenfalls dann nicht, wenn diese offiziellen Vorstellungen Formen der Objektivierung jener Wahrnehmungsund Handlungsschemata sind. Praktisch wirksam werden dann nicht die Regeln in ihrer expliziten Form, sondern die Dispositionen, die sich in diesen Regeln ausdrücken und wiederfinden (vgl. Bourdieu 1987: 198).

Damit ist die zweite Form von Regelskepsis bereits angesprochen, die Vermutung, dass die expliziten Regeln, die die Beteiligten selbst ihrem Verhalten zu Grunde liegen sehen, ebenfalls nicht das Erzeugungsprinzip der Regelmäßigkeiten ihrer Praktiken bilden. Die Begründung für diese Regelskepsis sieht Bourdieu darin, dass die Akteure, wenn sie "gegenüber ihrer Praxis eine Perspektive einzunehmen, die nicht mehr die der Aktion ist" (ebd.: 208), den gleichen Fehlschluss begehen, der dem Juridismus des Beobachters zu Grunde liegt: Die "Quasi-Theoretisierungen und Kodifizierungen" (ebd.: 213), die die Akteure in ihren offiziellen Diskursen perpetuieren, "verschleiern, selbst noch in ihren eigenen Augen, die Wahrheit ihrer praktischen Beherrschung als einer gelehrten Ignoranz (docta ignorantia), d.h. als eines praktischen Erkenntnismodus, der die Kenntnis seiner eigenen Prinzipien gerade nicht einschließt" (ebd.: 209). In diesem Sinne ist der offizielle Diskurs über die geltenden Regeln und die "allgemein gebilligten Vorstellungen" (Bourdieu 1987: 199) ein "Diskurs des betrogenen Betrügers" (Bourdieu 1979: 210). Er verleitet die Beteiligten und die Beobachter gleichermaßen, die Wirksamkeit expliziter Regeln bei der Hervorbringung der Regelmäßigkeiten des sozialen Lebens massiv zu überschätzen. Die Aufdeckung dieser beiderseitigen Fehlwahrnehmung führt dementsprechend zu den genannten beiden Formen der Regelskepsis.

\section{Kodifizierung und Kalkulierbarkeit}

Nun besitzen die im Modus praktischen Wissens und Könnens hervorgebrachten Handlungsformen allerdings eine Eigenschaft, die sich in bestimmten Situationen als problematisch oder gar hinderlich erweisen kann: das Merkmal der Unbestimmtheit und Unberechenbarkeit. "Der Habitus", so Bourdieu (1992: 101), "ist aufs engste mit dem Unscharfen und Ver- 
schwommenen verbunden. Als eine schöpferische Spontaneität, die sich in der unvorhergesehenen Konfrontation mit unaufhörlich neuen Situationen geltend macht, gehorcht er einer Logik des Unscharfen, Ungefähren". Man dürfe deshalb "in den Hervorbringungen des Habitus nicht nach mehr Logik suchen, als sie aufweisen: Die Logik der Praktik besteht darin, nicht weiter als bis zu jenem Punkt logisch zu sein, ab dem die Logik nicht mehr praktisch wäre." (ebd.: 102f.) Weil sich aber erst in der jeweils neuen Situation entscheidet, was es heißt, die Logik der Praktik praktisch werden zu lassen, was also die jeweils situationsangemessenen Hervorbringungen des Habitus sind, erweist sich die im praktischen Wissen und Können begründete Handlungskompetenz stets erst im Nachhinein. Sie wird erst als eine "retrospektive[] Notwendigkeit" (Bourdieu 1987: 106) sichtbar, nämlich als ein Handeln, "von dem es heißen wird, daß 'es nicht anders zu machen war'" (Bourdieu 1979: 225)

Jetzt ließe sich einwenden, dass dies eine sehr einseitige Betrachtung praktischen Wissens und Könnens sei, die ergänzt werden müsse um den Aspekt der Routinisierung, auf deren Grundlage die Praktiken dann eben doch prognostizierbar würden. Diesen Gesichtspunkt greift Bourdieu auf, indem er feststellt: "Daß man nun bestimmte Praktiken [...] voraussagen kann, gründet darin, daß Akteure mit dem entsprechenden Habitus sich auf eine ganz bestimmte Weise verhalten. Doch deshalb", so fährt er einschränkend fort, "beruht diese Tendenz, auf regelmäßige Weise zu handeln, und die dann, ist das Prinzip einmal explizit ausgebildet, zur Basis einer Prognose werden kann [...], nicht auf einer Regel oder einem ausdrücklich formulierten Gesetz." (Bourdieu 1992: 100f.) Mit anderen Worten: Die Basis der Prognose und das Erzeugungsprinzip der Regelmäßigkeit, die sie vorhersagen will, sind nicht identisch. Denn die Basis der Prognose ist die explizite Regel, welche die Regelmäßigkeit der Praxis nachträglich beschreibt, das Erzeugungsprinzip der Regelmäßigkeit dagegen ist ein praktisches Wissen und Können, dass eben nicht eine explizite Regel exekutiert. Die derart gewonnene Prognose ist also eine höchst trügerische, weil sie einfach nur eine in der Vergangenheit beobachtete Regelmäßigkeit in die Zukunft verlängert. Sie kann sich schnell als falsch erweisen, wenn in der neuen Situation die Abweichung von der bisherigen Regelmäßigkeit das Handeln das ist, von dem es heißen wird, dass es nicht anders zu machen war.

Es bleibt also dabei, dass "die vom Habitus erzeugten Verhaltensweisen [...] nicht die bestechende Regelmäßigkeit des von einem normativen Prinzip" oder einer sonstigen expliziten Regel "geleiteten Verhaltens 
aus[weisen]". Dies führt zu der Überlegung, dass es Situationen geben kann, in denen es sinnvoll oder gar notwendig ist, von habituell hervorgebrachtem auf regelgeleitetes, kodifiziertes Verhalten umzustellen, weil die Erwartungssicherheit des vom praktischen Wissen und Können gesteuerten Verhaltens dort nicht ausreicht. Das "Moment an Unbestimmtheit, Offenheit und Unsicherheit" des Habitus ließe sich etwa als Argument dafür werten, "daß man sich in kritischen, gefährlichen Situationen nicht vollständig ihm überlassen darf. Als allgemeine Gesetzmäßigkeit ließe sich formulieren, daß die Praktik ihrer Tendenz nach um so stärker kodifiziert ist, je gefährlicher die Situation ist. [...] Je gewaltträchtiger eine Situation ist, um so notwendiger ist es, durch Formgebung zu entschärfen; um so ratsamer ist es, das den Improvisationen des Habitus überlassene Verhalten durch ein Verhalten zu ersetzen, das durch ein systematisch gestiftetes, wenn nicht sogar kodifiziertes Ritual geregelt wird. Man braucht nur an die Sprache der Diplomatie oder die protokollarischen Regeln zu denken, die Vorrangigkeiten und Schicklichkeiten bei offiziellen Anlässen festlegen."

Ein anderes Beispiel ist der Straßenverkehr: "Wie im Zusammenhang mit dem Fahrverhalten zu sehen ist, erbringt das Aufstellen von Verkehrsregeln kollektive Vorteile der Klärung und Vereinheitlichung. Man weiß, woran man sich halten kann; man kann verläßlich prognostizieren, daß bei Kreuzungen alle von links kommenden Autos halten müssen. Die Kodifizierung mindert das Moment an Mehrdeutigkeit und Unschärfe besonders in Interaktionen. Sie erweist sich in all den Situationen als unabdingbar und wirksam, wo die Risiken des Zusammenstoßes, des Konflikts, des Unfalls und der Zufall [...] besonders hoch sind."

"Die Kodifizierung stellt einen grundlegenden Wandel dar, eine Veränderung des ontologischen Status, die sich vollzieht, sobald durch die Kodifizierung, eine juridische Tätigkeit, von in praktischem Zustand beherrschten [...] Schemata zu einem Kode, einer Grammatik übergegangen wird." "Kodieren heißt, mit dem Verschwommenen, Vagen, mit den unzulänglich gezogenen Grenzen und unscharfen Trennlinien Schluß zu machen [...] . Kodifiziert werden die Dinge klarer, einfacher, mitteilbarer". Und zugleich bringt die Kodifizierung "kollektive[] Vorteile in Gestalt von Kalkulierbarkeit und Vorhersehbarkeit [...] mit sich".

Wie soll man nun aus der praxistheoretischen Perspektive auf solche Überlegungen reagieren, die für bestimmte Situationen dem explizit regelgeleiteten Verhalten den Vorrang und Vorteil gegenüber dem praktischen Wissen und Können einräumt bzw. zumisst? Der spontane praxistheoretische 
Reflex ist vermutlich der, mit Regelskepsis zu reagieren. So, wie es Bourdieu (1998: 143ff.) beispielsweise gegen den Utilitarismus geltend macht, könnte man argumentieren, dass die "Reduktion auf das bewußte Kalkül" (ebd.: 144) auch hier eine irreführende Fehlwahrnehmung eines tatsächlich vielmehr auf dem "vorbewußten Einverständnis" des "praktischen Sinns", des "Sinns für das Spiel" beruhenden Wirkungszusammenhanges darstellt. Doch dieser Einwand greift zu kurz. Denn die angeführten Überlegungen entstammen keiner Forschungsrichtung, der man Praxisvergessenheit vorhalten könnte. Sie stammen vielmehr sämtlich von Bourdieu selbst (1992: 101, 104, 103, 106, 107).

Mit Bourdieu lässt sich also eine praxistheoretische Perspektive einnehmen, die zwei diametral entgegengesetzte Sichtweisen auf die Wirkungsweise und Wirksamkeit expliziter Regeln beinhaltet. Aus der regelskeptischen Perspektive sind explizite Regeln "Formen der Objektivierung der Wahrnehmungs- und Handlungsschemata" (Bourdieu 1987: 198), die nur als Ausdrucksformen der inkorporierten Dispositionen der Akteure wirksam werden, also kein eigenständiges Erzeugungsprinzip regelmäßigen Handelns sind. Auf dieser Sichtweise der expliziten Regel liegt in der Praxistheorie Bourdieus das Hauptgewicht. Zugleich erkennt er im Problem der Prognostizierbarkeit die Kehrseite einer Praxis, deren Regelhaftigkeit man sich stets erst retrospektiv versichern kann. Hier kommt - als Resultat der praxistheoretischen Analyse dieser Praxis - die andere Sichtweise auf explizite Regeln zum Tragen: Die Kodifizierung als Grundlage eines regelgeleiteten Handelns, das sich seiner verlässlichen Prognostizierbarkeit wegen in bestimmten Situationen gegenüber der impliziten Logik der Praxis durchsetzt.

\section{Bedingungen der Wirksamkeit expliziter Regeln}

Bourdieus Kriterien für Situationen, in denen Kodifizierungen wirksam und erforderlich sind, sind allerdings einerseits zu eng, andererseits zu weit gefasst. Die spezifische Gefährlichkeit der Situation ist ein zu enges Kriterium. Denn es lassen sich eine Vielzahl von Situationen benennen, die verhaltenswirksam explizit reguliert sind, ohne im Normalfall ein entsprechendes Gefährdungspotenzial zu besitzen: Man denke nur an die explizite Regelung von Ladenöffnungszeiten. Der Vorteil, als Kunde im Vorhinein zu wissen, wann man Einlass erhält und die Chance, bedient zu werden, ist evident, auch wenn die misslingende Handlungskoordination in diesem Fall normalerweise nicht besonders gefahrenträchtig ist. Umgekehrt ist die besondere 
Zufallsträchtigkeit ein zu weit gefasstes Kriterium. Denn die Fähigkeit des Umgangs mit Unerwartetem ist praxistheoretisch gesprochen ja gerade eine besondere Stärke der impliziten Logik der Praxis.

Es scheint mir deshalb sinnvoller, bei der spezifischen Differenz zwischen praktischem Wissen und Können und regelgeleitetem Handeln anzusetzen: der Differenz zwischen einer notwendig retrospektiven Orientierung einerseits und einer auf Prognostizierbarkeit zukünftiger Ereignisse gerichteten prospektiven Orientierung andererseits (vgl. Schulz-Schaeffer 1999: 414ff.). Ausgehend von dieser Differenz beruht die Chance, dass explizite Regeln erfolgreich verhaltenswirksam werden, erstens darauf, dass die an der Regel selbst ablesbaren Folgen der Regelbefolgung oder -verletzung tatsächlich hinreichend zuverlässig eintreten. Sie beruht zweitens darauf, dass diese Folgen von den Adressaten der Regel in einer Weise als erwünscht oder unerwünscht angesehen werden, die dazu führt, dass "das Interesse an ihrer Einhaltung stärker ist als das, sie zu mißachten" (Bourdieu 1992: 99). Drittens schließlich beruht sie darauf, dass die Adressaten wissen was es heißt, der betreffenden Regel zu folgen.

\subsection{Regel-Wirkungs-Zusammenhänge}

Regeln werden nicht schon dadurch wirksam, dass sie explizit formuliert werden - also nicht durch den Prozess der Kodifizierung allein -, sondern erst durch die Einrichtung von Regel-Wirkungs-Zusammenhängen. Solche Zusammenhänge von Regelorientierung und prognostizierbaren Wirkungen entsteht entweder dadurch, dass das regelgeleitete Verhalten selbst diese Wirkungen produziert. Oder aber die Wirkungen sind das Werk einer die Regelbefolgung überwachenden externen Instanz. Beispiele für RegelWirkungs-Zusammenhänge der letztgenannten Art sind die Rechtsvorschrift in Verbindung mit dem staatlichen Sanktionsapparat, die soziale Norm in Verbindung mit der Billigung oder Missbilligung durch eine relevante Bezugsgruppe oder die Lernanweisung in Verbindung mit der Erfolgskontrolle durch den Lehrer. In allen diesen Fällen geht es um die extern kontrollierte Orientierung an Sollensvorschriften, weshalb man von normativen RegelWirkungs-Zusammenhängen sprechen kann. Dagegen konstituieren RegelWirkungs-Zusammenhänge der erstgenannten Art den Bereich des Technischen: Hier sind die Regeln Anweisungen zum Hervorbringen von Ereignisketten mit bestimmten, im Vorhinein bekannten Wirkungen. Die Wirkungen werden also durch die Regelbefolgung selbst produziert. 
Bei normativen Regel-Wirkungs-Zusammenhängen sind die Regelbefolgung und die Herstellung der Regelfolgen typischerweise zwei unterschiedliche Aktivitäten. Die Verbindung beider Aktivitäten besteht darin, dass dem Adressaten der Regel bestimmte extern produzierte Wirkungen von einer Kontrollinstanz als Folgen seines Tuns gutgeschrieben bzw. aufgebürdet werden. Bei technischen Regel-Wirkungs-Zusammenhängen verhält es sich mit Blick auf den ersten, tendenziell aber auch mit Blick auf den zweiten Gesichtspunkt genau umgekehrt: Hier werden die fraglichen Wirkungen durch die Aktivität der Regelbefolgung selbst produziert. ${ }^{1}$ Aber nicht notwendigerweise sind diejenigen, die die Regeln befolgen, zugleich auch diejenigen, die von den Wirkungen profitieren oder sie zu erleiden haben. Vielmehr finden wir hier vielfältige Formen von Delegation - von stellvertretend für andere erfolgender Erzeugung von Wirkungen. Entscheidend sind dabei zwei Typen der Delegation (jeweils betrachtet aus der Perspektive dessen, der von den Wirkungen profitiert): die Delegation der Regelbefolgung an andere Akteure, die sich als Experten der Regelbefolgung der jeweiligen Art auszeichnen, und die Delegation an dingliche Arrangements, also an sachtechnische Artefakte, denen die Regel als Algorithmus mechanisch oder informationstechnisch eingeprägt ist.

\subsection{Interessen- und Durchsetzungskalküle}

Als zweite Bedingung dafür, dass explizite Regeln erfolgreich verhaltenswirksam werden, hatte ich das Interesse der Adressaten der Regel an der Realisierung oder Vermeidung der voraussehbaren Wirkungen ihres Regelgehorsams oder -ungehorsams benannt. Es reicht nicht aus, dass sichergestellt ist, dass die Wirkungen, wie sie sich auf der Grundlage des Regelwissens vorhersagen lassen, auch tatsächlich hinreichend zuverlässig eintreten. Eine zusätzliche Bedingung ist vielmehr, dass dieses Wissen über die zukünftigen Wirkungen gegenwärtigen Handelns oder Unterlassens Gegenstand eines rational zielgerichteten Handlungskalküls wird. Es lautet in seiner Grundform: Das Interesse an der Einhaltung der Regel ist dann größer als das gegenteilige Interesse, wenn die Vorteile des Regelgehorsams in Gestalt erwünschter Wirkungen die Nachteile des durch die Regel vorgeschriebenen Verhaltens überwiegen oder wenn umgekehrt die Nachteile des Regelungehorsams in

1. Vgl. zu dieser Differenz Habermas 1969: 63; ähnlich Linde 1972: 70, Linde 1982: 23 und, in der Beschreibungssprache der symmetrischen Anthropologie, Latour 1991: 111; 1992: 127; dazu insgesamt Schulz-Schaeffer 2000: 295ff. 
Gestalt unerwünschter Wirkungen die Vorteile überwiegen, die aus einer Handlungsalternative resultieren, welche die Missachtung der Regel voraussetzt. Aus der Perspektive der Etablierung und Durchsetzung regelgeleiteter Zusammenhänge führt dieses Interessenkalkül zu einem darauf aufbauenden Durchsetzungskalkül, das darin besteht, die Wirkungen eines RegelWirkungs-Zusammenhangs so einzurichten, dass die Kalküle der Adressaten der Regel zugunsten der Regelbefolgung ausgehen.

Dies alles ist bekannt und in der Sozialforschung schon vielfach expliziert worden. Man muss keine besondere Präferenz für zweckrationale Handlungserklärungen und Theorien rationaler Wahl zu haben, um die empirische Relevanz solcher Kalküle herauszustellen. Auch Bourdieu geht ganz selbstverständlich von der Wirksamkeit von Kalkülen dieser Art aus, wenn er argumentiert, dass ein Regelzusammenhang wie der der Straßenverkehrsordnung oder der des Sprachcodes sich deshalb "ohne größere Diskussion wie von selbst aufzwingt [...], weil er mit wenigen Ausnahmen zwischen relativ arbiträren Möglichkeiten entscheidet [...] und weil von keiner Seite größere Interessen im Spiel sind" (Bourdieu 1992: 107).

Hinzuzufügen ist, dass die Kalküle derer, die mit Blick auf die zu erwartenden Wirkungen über ihre Orientierung an der Regel entscheiden, und die derer, die mit Blick auf die angestrebte Regelhaftigkeit eines Zusammenhangs über Art der implementierten Wirkungen entscheiden, in der eben skizzierten unmittelbaren Weise nur dort greifen, wo die von der Regel prognostizierte Wirkung direkt auf die Adressaten der Regel zurückfällt. Dies ist, wie oben erwähnt, nur bei normativen Regel-WirkungsZusammenhängen der Normalfall. Im Fall technischer Regel-WirkungsZusammenhänge ist die Situation komplizierter. Unter der Bedingung der Ausdifferenzierung der Bereitstellung und der Nutzung technischer Wirkungen finden wir hier verschiedene, vermittelt (etwa über Nutzungsentgelte) aufeinander bezogene Interessen- bzw. Durchsetzungskalküle. Dieser Umstand bildet ein Einfallstor für andere als an der rein technischen Wirksamkeit orientierte Faktoren der Entscheidung über Technikentwicklung und nutzung.

\subsection{Situationsähnlichkeit}

Schließlich hatte ich noch eine dritte Bedingung dafür, dass explizite Regeln erfolgreich verhaltenswirksam werden, angesprochen: die Bedingung, dass die Adressaten wissen was es heißt, der betreffenden Regel zu folgen. Diese 
Bedingung, das ist seit dem späten Wittgenstein (1989: 287-290, 344f.) geläufig, ist nicht bereits dadurch erfüllt, dass die Adressaten die Regel kennen. In der Fassung Bourdieus lautet das Wittgenstein-Argument: "Es ist von Bedeutung, daß jeder Versuch [...] eine Praxis/Praktik auf der gehorsamen Erfüllung einer explizit formulierten Regel zu begründen, sich an der Frage nach den Regeln stößt, die die angemessenste Art und Weise [...] der Anwendung der Regel oder, wie man so schön sagt, der praktischen Umsetzung eines Repertoires an Vorschriften oder Techniken bestimmen, mit anderen Worten an der Frage nach der Kunst der Ausführung/Ausübung, worein sich, unausweichlich, der Habitus wieder einschleicht." (Bourdieu 1979: 203f.)

Hat also im Endeffekt doch die Praxis das letzte Wort? Ist es nur vordergründig so, dass die Regelmäßigkeiten bestimmter Ereignisabläufe das Werk regelgeleiteten Verhaltens sind? Wäre dies so, dann würde das Funktionieren von Regel-Wirkungs-Zusammenhängen, das sich zumindest im Bereich der vorhersehbaren Erzeugung technischer Wirkungen als empirischer Tatbestand kaum leugnen lässt, zu einem unerklärlichen Phänomen. Andererseits lässt sich aber auch am Wittgenstein-Argument nicht rütteln. Dessen empirische Relevanz hat sich beispielsweise am Scheitern der Begriffsjurisprudenz des 19. Jahrhunderts eindrucksvoll erwiesen, dem Versuch, ein rechtliches Regelsystem aufzustellen, dessen Regeln angewandt werden können, ohne ausgelegt werden zu müssen (vgl. Krawietz 1972).

Erforderlich ist deshalb eine Betrachtungsweise, die dem WittgensteinArgument Rechnung trägt und gleichzeitig erklärt, wie es möglich ist, expliziten Regeln in einer zielgerichtet prospektiven Weise zu folgen, also in einem Erkenntnismodus, der nicht der des stillschweigenden praktischen Wissens und Könnens ist. Der entscheidende Ausgangspunkt hierfür ist die Betrachtung der jeweiligen Situation, in der sich die Frage nach der richtigen Art und Weise der Anwendung der Regel stellt. Grundlage der praxistheoretischen Argumentation ist, dass praktisches Wissen und Können ein Erfordernis der "unvorhergesehenen Konfrontation mit unaufhörlich neuen Situationen" (vgl. oben) ist, für die explizite Regeln keine vollständigen Verhaltensanweisungen zu geben vermögen, weil sich das Unvorhersehbare eben nicht vorhersehen und deshalb auch nicht im Vorhinein explizit regeln lässt. In dieser Beobachtung liegt - umgekehrt gewendet - zugleich aber auch der Schlüssel zur Lösung des Problems: Was es heißt einer Regel zu folgen, lässt sich um so eindeutiger und vollständiger an der Regel selbst ablesen, je ähnlicher diejenige Situation, in der sich die Frage nach der Art und Weise 
ihrer Anwendung stellt, derjenigen Situation ist, deren Bedingungen die Regelvorschrift berücksichtigt.

Aus der Tatsache, dass die mit der Aufstellung expliziter Regeln verbundenen Regulierungsabsichten und -zwecke sich stets nur auf Vorhergesehenes richten kann, also nur auf diejenigen Umstände, die bei der Regelformulierung bewusst oder beiläufig im Blick waren, ergibt sich, dass jede solche Regel explizit oder implizit auf eine ideale oder zumindest typische Situation ihrer Anwendung bezogen ist: auf die Situation, in der die Regel ihr Höchstmaß an Vollständigkeit und Eindeutigkeit besitzt. Die Möglichkeit einer direkten Anwendung expliziter Regeln steigt und die Notwendigkeit, bezüglich der Frage ihrer Anwendbarkeit auf praktisches Wissen und Können rekurrieren zu müssen, sinkt dementsprechend in dem Maße, in dem die aktuelle Situation jener typischen Situation ähnlich ist. Der betreffende Regel-Wirkungs-Zusammenhang wird in der aktuellen Situation mithin in dem Maße wirksam, in dem er einen in der typischen Situation erfolgreichen Ablauf reproduziert.

Die Frage nach der Wirksamkeit von Regel-Wirkungs-Zusammenhängen stellt sich damit nun als Frage danach, wie eine solche Ähnlichkeit zwischen der typischen und der aktuellen Situation entsteht. Diesbezüglich sind zwei Vorgehensweisen zu beobachten: Die eine besteht darin, Situationsähnlichkeit gezielt herzustellen, die andere darin, autoritative Deutungen zu etablieren, die festlegen, wann und unter welchen Bedingungen eine Situation als eine Ausprägung der typischen Situation zu gelten hat.

Die Herstellung von Situationsähnlichkeit ist insbesondere bei technischen Regel-Wirkungs-Zusammenhängen bedeutsam und neben der Einrichtung des Regel-Wirkungs-Zusammenhangs selbst sicherlich die wichtigste Leistung der Produzenten technischer Wirkungen. Wie dies geschieht, ist von Latour am Beispiel des Milzbrand-Impfstoffs prägnant beschrieben worden. Die typische Situation, in der der von Pasteur entwickelte Wirkstoff seine gegen Milzbrand immunisierende Wirkung zuverlässig prognostizierbar entfaltet, ist die des Labors, in dem der Impfstoff entwickelt und erfolgreich getestet wurde. Die Antwort auf die Frage, wie es gelingt diesen Erfolg in den Viehställen auf dem Lande zu wiederholen, lautet Latours Analyse zufolge: "only by extending the laboratory itself. Pasteur cannot just hand out a few flasks of vaccine to farmers and say: 'OK, it works in my lab, get by with that.' If he were to do that, it would not work. The vaccination can work only on the condition that the farm [...] be in some crucial respects 
transformed according to the prescriptions of Pasteur's laboratory." (Latour 1983: 150f.)

Wie erfolgreich die menschlichen oder nichtmenschlichen Produzenten technischer Wirkungen zur Herstellung von Situationsähnlichkeit beitragen, lässt sich am Alltagsbeispiel der automobilen Fortbewegung gut illustrieren: Man nimmt den Fahrersitz ein, lässt den Motor an und sieht sich dann, egal ob man eine neue oder eine bekannte Wegstrecke zurückzulegen trachtet, normalerweise einer Situation gegenüber, die denen der früheren Benutzung hochgradig ähnlich ist: Eine Situation, in der einige wenige Entscheidungsalternativen im Vordergrund stehen: Erhöhung, Verringerung oder Beibehaltung der Geschwindigkeit, Veränderung oder Beibehaltung der Fahrtrichtung usw.

Im Fall normativer Regel-Wirkungs-Zusammenhänge geschieht die Erzeugung von Situationsähnlichkeit primär auf dem Wege der autoritativen Deutung von Situationen. In den Vorschriften des staatlichen Rechts erfolgt dies dadurch, dass im Text des Gesetzes die typischen Merkmale des jeweiligen Tatbestandes benannt werden, auf den sich das Ge- oder Verbot richtet. Die Verfügbarkeit einer solchen autoritativen Vorgabe hat dabei die Wirkung, dass der Rechtsanwender nicht sofort auf sein praktisches Wissen und Können verwiesen ist, sondern den fraglichen Vorgang zunächst auf Ähnlichkeit mit diesen typischen Tatbestandsmerkmalen hin betrachten kann.

\section{Die Abschirmung des technischen Kerns als Leistung der Praxis}

Zusammenfassend kann also festgehalten werden: Explizite Regeln werden als Mittel prospektiver Handlungsorientierung wirksam, wenn (1) die selbstsanktionierenden Effekte der Regelbefolgung oder die externen Sanktionsinstanzen hinreichend zuverlässig dafür sorgen, dass die Wirkungen so eintreffen, wie auf der Grundlage der Regel vorausgesagt; wenn (2) die Durchsetzungskalküle so auf die Interessenkalküle der Beteiligten bezogen sind, dass diese sich von den zu erwartenden Wirkungen zu Regeltreue motivieren lassen; und wenn (3) die technisch erzeugte oder autoritativ festgelegte Situationsähnlichkeit dafür sorgt, dass es hinreichend Gelegenheiten gibt, die Regeln unproblematisch und ohne größeren Interpretationsbedarf anzuwenden. Diese drei Faktoren konstituieren zusammengenommen das, was ich mit einem Begriff von Thompson (1967: 19ff.) als den technischen Kern eines Regel-Wirkungs-Zusammenhangs bezeichnen möchte. Die Überlegungen 
des vorangegangenen Abschnitts lassen sich mithin dahingehend zusammenfassen, dass Kodifizierungen dann die Chance haben, verhaltenswirksam zu werden, wenn es gelingt, technische Kerne dieser Art zu etablieren.

Thompson geht es mit dem Konzept des technischen Kerns um eine Synthese zweier konkurrierender organisationstheoretischer Perspektiven: der Betrachtung der Organisation als eines Instruments rational geplanter Zielerreichung einerseits und dem Verständnis der Organisation als eines ungeplant evolutionären Zusammenhangs von Elementen andererseits, der durch spontane Anpassung an die unvorhergesehenen und unkontrollierbaren Ereignissen der Umwelt überlebt und sich entwickelt (vgl. ebd.: 4-8). Sein Synthesevorschlag lautet, beide Aspekte als wesentlich für die rational zielverfolgende Organisation zu betrachten. Diese ist demnach einerseits bestrebt, zum Zweck planbarer Zielerreichung einen Bereich kontrollierter Ursache-Wirkungs-Zusammenhänge zu etablieren, den technischen Kern eben. In einer Umwelt, die sie in unvorhersehbarer Weise mit externen Anforderungen konfrontiert, muss sie andererseits dafür sorgen, dass diese externen Faktoren nicht störend auf den technischen Kern durchschlagen. Die Organisation muss also in der Lage sein, adaptiv und flexibel auf Umweltanforderungen zu reagieren, und zwar in einer Weise, die den technischen Kern gegen Turbulenzen der Umwelt abpuffert. Die Effizienz der Organisation mit Blick auf die Zielerreichung gründet mithin nicht allein in der Effizienz des technischen Kerns, sondern ebenso in der Fähigkeit, unerwartete Ereignisse in der Organisationsumwelt, durch flexiblen und adaptiven Umgang mit ihnen, vom technischen Kern fernzuhalten (vgl. ebd.: 19ff.).

Was Thompson hier beschreibt, lässt sich allgemeiner fassen als das $\mathrm{Zu}$ sammenwirken eines Bereichs regelgeleiteten Verhaltens und eines ihn umgebenden Bereichs praktischen Wissens und Könnens, verbunden mit der These, dass die Wirksamkeit des ersten Bereichs, des technischen Kerns, nicht allein auf der Einrichtung regelgeleiteter Abläufe beruht, sondern ebenso darauf, dass der umgebende Bereich praktischen Wissens und Könnens ihn gegen die unvorhersehbaren Anforderungen neuer Situationen abschirmt. Damit löst sich nun auch das im vorigen Abschnitt benannte Paradox auf, wie es gleichzeitig möglich ist, das Wittgenstein-Argument vom unendlichen Regelregress anzuerkennen, und dem empirischen Tatbestand des Funktionierens von Regel-Wirkungs-Zusammenhängen Rechnung zu tragen.

Für sich genommen, ist der Verweis auf die technische Herstellung oder autoritative Erzeugung von Situationsähnlichkeit als Lösung für das An- 
wendungsproblem expliziter Regeln nicht vollständig befriedigend. Bestimmte Probleme der Anwendbarkeit einer Regel werden auf diese Weise zwar gelöst. Die Frage nach dem richtigen Umgang mit einem RegelWirkungs-Zusammenhang, der die Situationsähnlichkeit herstellt oder definiert, stellt sich im Zweifelsfall dennoch. Denn die Häufung von Festlegungen auf Festlegungen, so das Wittgenstein-Argument, führt eben nie zu einem Ende.

Man nimmt beispielsweise wieder den Fahrersitz des Autos ein, lässt den Motor an und sieht sich mit Blick auf Fragen der Veränderung von Fahrtrichtung und -geschwindigkeit einer Situation unproblematischer Anwendbarkeit der einschlägigen Benutzungsregeln gegenüber. Aber nun stellt sich das Problem, in eine Parklücke ein- bzw. aus ihr auszuparken. Auch für diesen Zweck wird in der Fahrschule Regelwissen vermittelt. Aber nun ist jede Parklücke anders, mal kürzer, mal länger, mal breiter mal schmaler usw. Trotz aller Bemühungen um die technische Herstellung von Situationsähnlichkeit ist die Situation in gewissem Umfang also stets eine neue Situation. Um den technischen Regel-Wirkungs-Zusammenhang wirksam zum Zuge kommen zu lassen, hilft weiteres Regelwissen also nur begrenzt weiter. Man muss das Ein- oder Ausparken vielmehr im Gefühl haben, als inkorporiertes praktisches Wissen und Können im Umgang mit je neuen Situationen besitzen, um nicht endlos hin- und herzurangieren.

Gleiches gilt entsprechend für die autoritative Erzeugung von Situationsähnlichkeit, wie sie bei normativen Regel-Wirkungs-Zusammenhängen im Vordergrund steht. So definiert beispielsweise der ehemalige $\S 847$, Abs. 1 des Bürgerlichen Gesetzbuches die Situationen, in denen jemand Schmerzensgeld einklagen kann, wie folgt: "Im Falle der Verletzung des Körpers oder der Gesundheit sowie im Falle der Freiheitsentziehung kann der Verletzte auch wegen des Schadens, der nicht Vermögensschaden ist, eine billige Entschädigung in Geld verlangen." Sofern eine entsprechende Situation vorliegt, ist die Angelegenheit also relativ eindeutig. Müssten dann aber nicht auch andere immaterielle Schädigungen als Beeinträchtigung der persönlichen Freiheit behandelt werden, für die Schmerzensgeld beansprucht werden kann: die nicht autorisierte Bezugnahme auf Personen zu Werbezwecken etwa oder das Mobbing am Arbeitsplatz? Auch diese Fragen werden nicht durch die Anwendung von Regeln beantwortbar, sondern durch Verweis auf gemeinsame Praktiken, nämlich auf die richterliche Spruchpraxis und die Praxis der rechtswissenschaftlichen Gesetzesauslegung. Und die 
sich in diesen Praktiken herausbildende Regelmäßigkeit der Beurteilung entsprechender Fälle, bejaht diese Fragen (Wesel 1979: 88f.). ${ }^{2}$

Das gleiche Zusammenspiel von explizit regelgeleitetem Handeln und handlungspraktischen Dispositionen bildet auch im Bereich der Interessenund Durchsetzungskalküle, also der zweiten von mir benannten Bedingung der Verhaltenswirksamkeit von Kodifizierungen, den Rahmen. Die Feststellung, dass es im Umgang mit Regel-Wirkungs-Zusammenhängen zweckbezogene Interessenkalküle sind, durch die das Wissen über zukünftig zu erwartende Wirkungen für das gegenwärtige Handeln relevant wird, steht nicht in unüberbrückbarem Widerspruch zu der praxistheoretischen Beobachtung, wonach "[s]oziale Akteure, die den Sinn für das Spiel besitzen und die Unzahl der praktischen Wahrnehmungs- und Bewertungsschemata inkorporiert haben, die [...] als Prinzipien der Wahrnehmung und Gliederung der Welt (fungieren), [...] die Ziele ihrer Praxis nicht als Zwecke zu setzen (brauchen)." (Bourdieu 1998: 144) Vielmehr bewirken die praktischen Dispositionen, dass aus dem unermesslichen Raum möglicher Wünsche und Ziele der größte Teil von vornherein als undenkbare oder nicht wünschenswerte ausgeschieden werden bzw. gar nicht erst in den Horizont des Wahrnehmbaren gelangen (Bourdieu 1987: 100). Und dies ist die Voraussetzung dafür, dass die verbleibenden Interessen und Ziele um so eindeutiger identifizierbar werden und den Handlungskalkülen um so berechenbarer zu Grunde gelegt werden können. Auch in dieser Hinsicht haben die praktischen Denk- und Wahrnehmungsschemata mit Blick auf den technischen Kern von Regel-Wirkungs-Zusammenhängen die Funktion der Abschirmung gegen unvorhersehbare Störungen. In diesem Fall geht es dabei um die Abschirmung gegen Störungen, die sich aus plötzlichen Veränderungen der Interessen der Adressaten expliziter Regeln ergeben würden, die dann gegebenenfalls die Durchsetzungskalküle unwirksam machen und damit auch den Regel-Wirkungs-Zusammenhang selbst gefährden würden.

Insgesamt lässt sich also ganz im Sinne des Wittgenstein-Arguments festhalten, dass Regel-Wirkungs-Zusammenhänge als Ressourcen prospektiven Handelns nur unter der Bedingung wirksam werden können, dass

2. Zu ergänzen ist, dass in einer Neufassung (vom 19.07.2002) des Ausgleichs für immaterielle Schäden im jetzigen $\S 253$, Abs. 2 des BGB dieser extensiven Auslegung des Begriffs "Freiheitsentziehung" in der Rechtspraxis dadurch Rechnung getragen wird, dass sich an seiner Stelle nun die allgemeinere Formulierung "Verletzung der Freiheit" findet. Auf eine eigenständige Kodifizierung eines Schadensausgleichs für die Verletzung von Persönlichkeitsrechten ist allerdings verzichtet worden. Manches überlässt man eben besser der Praxis als es explizit zu regulieren. 
zugleich alle jene Gepflogenheiten der Praxis als selbstverständlich vorausgesetzt werden können, die die verbleibende Uneindeutigkeit und Unvollständigkeit stillschweigend beseitigt, die jede explizite Regel aufweist, sobald sie auf eine Situation trifft, deren Eintreten bei ihrer Formulierung nicht berücksichtigt wurde. Es ist mithin nur deshalb möglich, einen bestimmten Ausschnitt des Handelns explizit zu regeln, weil alles übrige Handeln, das in der einen oder anderen Weise mit dem reglementierten Ausschnitt zusammenhängt, der abgestimmten Improvisation gemeinsamer Dispositionen überlassen werden kann.

Dabei ist wichtig zu betonen, dass dieses Ergebnis nicht identisch ist mit der regelskeptischen Position innerhalb der Praxistheorie, wonach RegelmäBigkeiten des sozialen Leben nur scheinbar regelgeleitet zu Stande kommen, tatsächlich aber der impliziten Logik der Praxis folgen. Innerhalb des technischen Kerns von Regel-Wirkungs-Zusammenhängen ist die Orientierung an expliziten Regeln das Erzeugungsprinzip des geregelten Ablaufs und genau dies konstituiert die spezifische Differenz solcher Arrangements zu den Regelmäßigkeiten gemeinsamer Praktiken: die Möglichkeit, mit Blick auf zukünftig zu erwartende Wirkungen zu handeln. Zugleich wird diese Möglichkeit stets erkauft durch eine Abhängigkeit von allen jenen Praktiken, die den technischen Kern abschirmen. Und in dem Maße, in dem dies der Fall ist, steht und fällt die Wirksamkeit prospektiven Handelns weiterhin mit den immer erst im Rückblick erkennbaren Regelmäßigkeiten gemeinsamer Praktiken.

\section{Ausblick}

Die voranstehenden Überlegungen verdeutlichen, dass sich auch im Rahmen des praxistheoretischen Paradigmas eine Position vertreten lässt, derzufolge explizite Regeln eigenständig verhaltenswirksam werden können. Abschließend soll überlegt werden, wie diese Position sich mit der grundlegenden Regelskepsis vereinbaren lässt, die, wie eingangs dargestellt, für die Entwicklung des praxistheoretischen Gedankens zunächst von so zentraler Bedeutung war. Eine brauchbare Antwort ergibt sich meiner Meinung nach, wenn man die Situation, in der die Regelmäßigkeiten des Handelns ausschließlich aus der "abgestimmten Improvisation der gemeinsamen Dispositionen" (Bourdieu 1979: 215) resultiert, und die Situation, in der die Regelhaftigkeit des Handelns ausschließlich dem an Regel-WirkungsZusammenhängen orientierten rationalen Kalkül entspringt, als die beiden 
idealtypischen Pole eines Kontinuums betrachtet. Und es spricht vieles dafür, dass in der empirischen Realität keiner dieser beiden Pole jemals ganz erreicht wird.

Für den Bereich des regelorientierten Handelns gilt dies bereits aufgrund des Erfordernisses der handlungspraktischen Abschirmung des technischen Kerns. Aber auch in anderer Hinsicht werden die kodifiziert objektvierten Regel-Wirkungs-Zusammenhänge vielfältig ergänzt oder überlagert durch Denk-, Wahrnehmungs- und Handlungsschemata, die sie dann zugleich zu Ausdrucksformen des Habitus im "opus operatum" (Bourdieu 1987: 98) machen. So betont schon Weber, dass die prospektive Handlungsorientierung, die ein staatlich garantiertes und sanktioniertes Recht ermöglicht, keineswegs zwangsläufig zur Folge hat, "daß etwa diejenigen, welche sich der Ordnung [...] fügen, dies vorwiegend oder auch nur überhaupt um deswillen tun, weil ein Zwangsapparat [...] dafür zur Verfügung steht. Davon ist", so Weber, "keine Rede." (Weber $1972<1922>$ : 183) Vielmehr gelte: "Die breiten Schichten der Beteiligten verhalten sich der Rechtsordnung entsprechend, entweder weil die Umwelt dies billigt und das Gegenteil nicht billigt, oder nur aus dumpfer Gewohnheit an die als Sitte eingelebten Regelmäßigkeiten des Lebens" (ebd.: 182). Letzteres aber bedeutet im Sinne der Bourdieu'schen Dialektik von einverleibter und objektivierter Geschichte, dass die Rechtsordnung dann als das in Worten objektivierte Gegenstück inkorporierter Dispositionen vermittels dieser Dispositionen wirksam wird (vgl. Bourdieu 1987: 98, 106f., 198f.).

Ein bekanntes Beispiel für solche Überlagerungen der prospektiven Regelorientierung durch Praxisschemata aus dem Bereich technischer RegelWirkungs-Zusammenhänge ist die so genannte Qwerty-Tastaur der Schreibmaschine und jetzt des Computers. Als Qwerty-Tastatur wird die spezifische Buchstabenanordnung auf der Tastatur dieser Schreibgeräte bezeichnet, die sich seit den frühen Tagen der Remington-Schreibmaschine bis heute mit nur kleinen Veränderungen in der ursprünglichen Form erhalten hat. Ginge es allein nach der expliziten Logik der Einrichtung möglichst wirksamer Regel-Wirkungs-Zusammenhänge, dann hätte diese Buchstabenanordnung längst einer ergonomischeren Anordnung, etwa der des "Dvorak Simplified Keyboard" weichen müssen (vgl. Diamond 1997). Tatsächlich aber wirkt sie als das gegenständlich objektivierte Gegenstück des inkorporierten Maschineschreiben-Könnens bis heute fort. ${ }^{3}$

3. Zur symbolischen Überlagerung von Techniknutzung vgl. Schulz-Schaeffer 2002: 48ff. 
Auf der anderen Seite ist es aber auch hochgradig unwahrscheinlich, dass es regelmäßige Handlungspraktiken geben kann, die nicht zugleich auch in einem gewissen Umfang in regelgeleitetes Handeln umschlagen. Offensichtlich neigen bereits die am stärksten durch gemeinsame Praktiken geregelten Gesellschaften oder gesellschaftlichen Bereiche dazu, die Regelmäßigkeiten ihrer Praktiken auf dem Wege der sekundären Interpretation als regelhaft zu explizieren. Und wenn auch diese Regeln als nachträgliche Rationalisierung gemeinsamer Praktiken auf die Welt kommen, so heißt dies keineswegs, dass sie nur auch solche in der Welt bleiben. "Die Illusion des Juridismus", so Bourdieu (1992: 110), "drängt sich nicht nur dem Forscher auf. Sie wirkt in der Realität selbst. Und so muß eine adäquate Theorie der Praktik sie auch berücksichtigen." Mit anderen Worten: Die Beschreibung, die die Regelmäßigkeiten einer gemeinsamen Praxis regelhaft abbildet, ist zunächst in der Tat nichts anderes als deren sekundäre Interpretation und nachträgliche Rationalisierung. Sobald allerdings das explizierte Wissen um diese Regelhaftigkeit handlungswirksam wird, kommt etwas Neues hinzu: Nun gibt es Akteure, die sich aufgrund dieser Regel und nicht allein aufgrund eingelebter Gewohnheit handeln. Und in dem Maß, in dem das der Fall ist, wird die Regel dann doch zum Erzeugungsprinzip der Praxis, die sie zuerst nur nachträglich rationalisiert hatte, und aus der anfänglichen Illusion wird empirische Realität.

Die Berechtigung der regelskeptischen Annahmen der Praxistheorie im Verhältnis zur Berechtigung der gegenteiligen Annahme einer eigenständigen Verhaltenswirksamkeit expliziter Regeln ergibt sich mithin nur im $\mathrm{Zu}-$ sammenhang mit der Beantwortung der Frage, wo auf dem Kontinuum zwischen den beiden Polen der jeweils betrachtete empirische Fall anzusiedeln ist. In jedem Fall aber ist die plausibelste Anfangshypothese die, mit einem gewissen Maß an eigenständiger Wirksamkeit expliziter Regeln auch dort zu rechnen, wo diese zunächst nur nachträgliche Rationalisierungen zu sein scheinen, und umgekehrt die Wirksamkeit praktischen Wissens und Könnens in einem gewissen Umfang auch dort in Rechnung zu stellen, wo man auf zweckbezogen eingerichtete Regel-Wirkungs-Zusammenhänge trifft.

\section{Literatur}

Bourdieu, Pierre (1979): Entwurf einer Theorie der Praxis auf der ethnologischen Grundlage der kabylischen Gesellschaft, Frankfurt/Main:

Suhrkamp. 
Bourdieu, Pierre (1981): "Men and Machines", in: Knorr-Cetina, Karin/Cicourel, Aaron V. (Hg.), Advances in Social Theory and Methodology. Toward an Integration of Micro- and Macro-Sociologies, Boston: Routledge \& Kegan Paul, S. 304-317.

Bourdieu, Pierre (1987): Sozialer Sinn. Kritik der theoretischen Vernunft, Frankfurt/Main: Suhrkamp.

Bourdieu, Pierre (1992): Rede und Antwort, Frankfurt/Main: Suhrkamp.

Bourdieu, Pierre (1998): Praktische Vernunft. Zur Theorie des Handelns, Frankfurt/Main: Suhrkamp.

Diamond, Jared (1997): "The Curse of QWERTY", in: Discover Magazine $18, \mathrm{~S}$.

Habermas, Jürgen (1969): Technik und Wissenschaft als 'Ideologie', Frankfurt/Main: Suhrkamp.

Krawietz, Werner (1972): "Juristische Methodik und ihre rechtstheoretischen Implikationen", in: Albert, Hans/Luhmann, Niklas/Maihofer, Werner/Weinberger, Ota (Hg.), Rechtstheorie als Grundlagenwissenschaft der Rechtswissenschaft. Jahrbuch für Rechtssoziologie und Rechtstheorie, Bd. 2, Düsseldorf: Bertelsmann, S. 12-42.

Latour, Bruno (1983): "Give Me a Laboratory and I will Raise the World", in: Knorr-Cetina, Karin/Mulkay, Michael (Hg.), Science Observed. Perspectives on the Social Study of Science, London u.a.: Sage Publications, S. 141-170.

Latour, Bruno (1991): "Technology is Society Made Durable", in: Law, John (Hg.), A Sociology of Monsters: Essays on Power, Technology and Domination, London u.a.: Routledge, S. 103-131.

Latour, Bruno (1992): "Where are the Missing Masses? The Sociology of a Few Mundane Artifacts", in: Bijker, Wiebe E./Law, John (Hg.), Cambridge, Mass. u.a.: The MIT Press, S. 225-258.

Linde, Hans (1972): Sachdominanz in Sozialstrukturen, Tübingen: J. C. B. Mohr.

Linde, Hans (1982): "Soziale Implikationen technischer Geräte, ihrer Entstehung und Verwendung", in: Jokisch, Rodrigo (Hg.), Techniksoziologie, Frankfurt/Main: Suhrkamp, S. 1-31.

Schulz-Schaeffer, Ingo (1999): "Technik und die Dualität von Ressourcen und Routinen", in: Zeitschrift für Soziologie 28, S. 409-428.

Schulz-Schaeffer, Ingo (2000): Sozialtheorie der Technik, Frankfurt/Main u.a.: Campus Verlag.

Schulz-Schaeffer, Ingo (2002): "Technik als altes Haus und als geschichtsloses Appartement. Vom Nutzen und Nachteil der Praxistheorie Bourdieus für die Techniksoziologie", in: Ebrecht, Jörg/Hillebrandt, Frank (Hg.), Bourdieus Theorie der Praxis. Erklärungskraft - Anwendung - Perspektiven, Wiesbaden: Westdeutscher Verlag, S. 4765.

Thompson, James D. (1967): Organizations in Action. Social Science Bases of Administrative Theory, NewYork u.a.: McGraw. 
Weber, Max $(1972<1922>)$ : Wirtschaft und Gesellschaft. Grundriß der verstehenden Soziologie, 5., revidierte Aufl., besorgt von Johannes Winckelmann, Tübingen: Mohr.

Wesel, Uwe (1979): "hM", in: Enzensberger, Hans Magnus (Hg.), Kursbuch 56: Unser Rechtsstaat, Berlin: Kursbuch/Rotbuch-Verl., S. 88-109.

Wittgenstein, Ludwig (1989): Tractatus logico-philosophicus, Tagebücher 1914-1916, Philosophische Untersuchungen. Werkausgabe. Band 1., Frankfurt/Main: Suhrkamp. 